A NNALES

UNIVERSITATIS MARIAE CURIE-SKŁODOWSKA

LUBLIN - POLONIA

E. A. OYEKAN and T. O. OPOOLA

\title{
On a subordination result for analytic functions defined by convolution
}

ABSTRACT. In this paper we discuss some subordination results for a subclass of functions analytic in the unit disk $U$.

1. Introduction. Let $A$ be the class of functions $f(z)$ analytic in the unit disk $U=\{z:|z|<1\}$ and normalized by

$$
f(z)=z+\sum_{n=2}^{\infty} a_{n} z^{n} .
$$

We denote by $K(\alpha)$ the class of convex functions of order $\alpha$, i.e.,

$$
K(\alpha)=\left\{f \in A: \operatorname{Re}\left(1+\frac{z f^{\prime \prime}(z)}{f^{\prime}(z)}\right)>\alpha, z \in U\right\} .
$$

Definition 1 (Hadamard product or convolution). Given two functions $f(z)$ and $g(z)$, where $f(z)$ is defined in (1.1) and $g(z)$ is given by

$$
g(z)=z+\sum_{n=2}^{\infty} b_{n} z^{n},
$$

2000 Mathematics Subject Classification. 30C45, 30C80.

Key words and phrases. Subordination, analytic functions, Hadamard product (convolution). 
the Hadamard product (or convolution) $f * g$ of $f(z)$ and $g(z)$ is defined by

$$
(f * g)(z)=z+\sum_{n=2}^{\infty} a_{n} b_{n} z^{n}=(g * f)(z) .
$$

Definition 2 (Subordination). Let $f(z)$ and $g(z)$ be analytic in the unit disk $U$. Then $f(z)$ is said to be subordinate to $g(z)$ in $U$ and we write

$$
f(z) \prec g(z), \quad z \in U,
$$

if there exists a Schwarz function $w(z)$, analytic in $U$ with $w(0)=0,|w(z)|<$ 1 such that

$$
f(z)=g(w(z)), \quad z \in U .
$$

In particular, if the function $g(z)$ is univalent in $U$, then $f(z)$ is subordinate to $g(z)$ if

$$
f(0)=g(0), \quad f(U) \subseteq g(U) .
$$

Definition 3 (Subordinating factor sequence). A sequence $\left\{b_{n}\right\}_{n=1}^{\infty}$ of complex numbers is said to be a subordinating factor sequence if whenever $f(z)$ of the form (1.1) is analytic, univalent and convex in $U$, the subordination is given by

$$
\sum_{n=1}^{\infty} a_{n} b_{n} z^{n} \prec f(z), \quad z \in U, a_{1}=1 .
$$

We have the following theorem.

Theorem 1.1 (Wilf [5]). The sequence $\left\{b_{k}\right\}_{k=1}^{\infty}$ is a subordinating factor sequence if and only if

$$
\operatorname{Re}\left\{1+2 \sum_{k=1}^{\infty} b_{k} z^{k}\right\}>0, \quad z \in U .
$$

Let

$$
M(\alpha)=\left\{f \in A: \operatorname{Re}\left(\frac{z f^{\prime}(z)}{f(z)}\right)<\alpha, z \in U\right\}
$$

and let

(1.7) $M^{\delta}(b, \delta)=\left\{f \in A: \operatorname{Re}\left\{1-\frac{2}{5}+\frac{2 D^{\delta+2} f(z)}{b D^{\delta+1} f(z)}\right\}<\alpha, \alpha>0, z \in U\right\}$. 
Here $D^{\delta} f(z)$ is the Ruschewey's derivative defined as

$$
\begin{aligned}
D^{\delta} f(z) & =\frac{z}{(1-z)^{\delta+1}} * f(z) \\
& =\left(z+\sum_{n=2}^{\infty} \frac{\Gamma(n+\delta)}{(n-1) ! \Gamma(1+\delta)}\right) *\left(z+\sum_{n=2}^{\infty} a_{n} z^{n}\right) \\
& =z+\sum_{n=2}^{\infty} \frac{\Gamma(n+\delta)}{(n-1) ! \Gamma(1+\delta)} a_{n} z^{n}, \quad \delta \geq-1 .
\end{aligned}
$$

Theorem 1.2 ([3]). If $f(z) \in A$ satisfies

$$
\begin{aligned}
& \sum_{n=2}^{\infty}\{|b(1-k)(\delta+2)+2(n-1)|+\mid b(1-2 \alpha+k)(\delta+2) \\
& \quad+2(n-1) \mid\} \frac{\Gamma(n+\delta+1)}{(n-1) ! \Gamma(3+\delta)}\left|a_{n}\right| \leq 2|b(1-\alpha)|
\end{aligned}
$$

where $b$ is a non-zero complex number, $\delta \geq-1,0 \leq k \leq 1$ and $\alpha>1$, then $f(z) \in M^{\delta}(b, \alpha)$.

It is natural to consider the class $M^{\delta^{*}}(b, \alpha) \subset M^{\delta}(b, \alpha)$ such that

$$
\begin{aligned}
& M^{\delta^{*}}(b, \alpha)=\left\{f \in A: \sum_{n=2}^{\infty}\{|b(1-k)(\delta+2)+2(n-1)|\right. \\
& \quad+|b(1-2 \alpha+k)(\delta+2)+2(n-1)|\} \frac{\Gamma(n+\delta+1)}{(n-1) ! \Gamma(3+\delta)}\left|a_{n}\right| \\
& \quad \leq|b(1-\alpha)|\} .
\end{aligned}
$$

Our main result in this paper is the following theorem.

Theorem 1.3. Let $f \in M^{\delta^{*}}(b, \alpha)$, then

$$
\frac{B}{C}(f * g)(z) \prec g(z)
$$

where

$$
\begin{aligned}
& B=|b(1-k)(\delta+2)+2|+|b(1-2 \alpha+k)(\delta+2)+2| \\
& C=2[2|b(1-\alpha)|+|b(1-k)(\delta+2)+2|+|b(1-2 \alpha+k)(\delta+2)+2|],
\end{aligned}
$$

$\delta \geq-1,0 \leq k \leq 1, b$ is a non-zero complex number and $g(z) \in K(\alpha)$, $z \in U$. Moreover,

$$
\operatorname{Re}(f(z))>-\frac{C}{2 B}
$$

The constant factor

$$
\frac{B}{C}=\frac{|b(1-k)(\delta+2)+2|+|b(1-2 \alpha+k)(\delta+2)+2|}{2[2|b(1-\alpha)|+|b(1-k)(\delta+2)+2|+|b(1-2 \alpha+k)(\delta+2)+2|]}
$$


cannot be replaced by a larger one.

2. Proof of the main result. Let $f(z) \in M^{\delta^{*}}(b, \alpha)$ and suppose that

$$
g(z)=z+\sum_{n=2}^{\infty} b_{n} z^{n} \in K(\alpha)
$$

Then by definition,

$$
\frac{B}{C}(f * g)(z)=\frac{B}{C}\left(z+\sum_{n=2}^{\infty} a_{n} b_{n} z^{n}\right) .
$$

Hence, by Definition 3, to show the subordination (1.10) it is enough to prove that

$$
\left\{\frac{B}{C} a_{n}\right\}_{n=1}^{\infty}
$$

is a subordinating factor sequence with $a_{1}=1$. Therefore, by Theorem 1.1 it is sufficient to show that

$$
\operatorname{Re}\left\{1+2 \sum_{n=1}^{\infty} \frac{B}{C} a_{n} z^{n}\right\}>0, \quad z \in U
$$

Now,

$$
\begin{aligned}
\operatorname{Re}\left\{1+2 \sum_{n=1}^{\infty} \frac{B}{C} a_{n} z^{n}\right\} & =\operatorname{Re}\left\{1+2 \frac{B}{C} a_{1} z+\frac{2}{C} \sum_{n=2}^{\infty} B a_{n} z^{n}\right\} \\
& \geq 1-2 \frac{B}{C} r-\frac{2}{C} \sum_{n=2}^{\infty} B\left|a_{n}\right| r^{n} .
\end{aligned}
$$

Since $\frac{\Gamma(n+\delta+1)}{(n-1) ! \Gamma(3+\delta)}$ is a monotone non-decreasing function of $n=2,3, \ldots$, we have

$$
\begin{aligned}
& \operatorname{Re}\left\{1+2 \sum_{n=1}^{\infty} \frac{B}{C} a_{n} z^{n}\right\}>1-2 \frac{B}{C} r \\
& -\frac{2}{C} \sum_{n=2}^{\infty}\{[\mid b(1-k)(\delta+2)+2(n-1)]|+| b(1-2 \alpha+k)(\delta+2)+2(n-1) \mid\} \\
& \quad \times \frac{\Gamma(n+\delta+1)}{(n-1) ! \Gamma(3+\delta)}\left|a_{n}\right| r, \quad 0<r<1 .
\end{aligned}
$$

By (1.8)

$$
\begin{aligned}
\sum_{n=2}^{\infty}\{\mid b(1-k)(\delta+2) & +2(n-1)|+| b(1-2 \alpha+k)(\delta+2)+2(n-1) \mid\} \\
& \times \frac{\Gamma(n+\delta+1)}{(n-1) ! \Gamma(3+\delta)}\left|a_{n}\right| \leq 2|b(1-\alpha)| .
\end{aligned}
$$


Hence,

$$
\begin{aligned}
\operatorname{Re}\left\{1+2 \sum_{n=1}^{\infty} \frac{B}{C} a_{n} z^{n}\right\} & =\operatorname{Re}\left\{1+2 \frac{B}{C} a_{1} z+\frac{2}{C} \sum_{n=2}^{\infty} B a_{n} z^{n}\right\} \\
& >1-2 \frac{B}{C} r-\frac{4|b(1-\alpha)|}{C} r \\
& =1-\frac{2 B+4|b(\alpha-1)|}{C} r \\
& =1-r>0
\end{aligned}
$$

$(|z|=r<1)$. Therefore, we obtain

$$
\operatorname{Re}\left\{1+2 \sum_{n=1}^{\infty} \frac{B}{C} a_{n} z^{n}\right\}>0
$$

which is (2.3) that was to be established.

We now show that

$$
\operatorname{Re}(f(z))>-\frac{C}{2 B}
$$

Taking

$$
g(z)=\frac{z}{1-z} \in K(\alpha)
$$

(1.10) becomes

$$
\frac{B}{C} f(z) \prec \frac{z}{1-z} .
$$

Therefore,

$$
\operatorname{Re}\left(\frac{B}{C} f(z)\right)>\operatorname{Re}\left(\frac{z}{1-z}\right)
$$

Since

$$
\operatorname{Re}\left(\frac{z}{1-z}\right)>-\frac{1}{2}, \quad|z|<r
$$

this implies that

$$
\frac{B}{C} \operatorname{Re}(f(z))>-\frac{1}{2} .
$$

Hence, we have

$$
\operatorname{Re}(f(z))>-\frac{C}{2 B}
$$

which is (1.11).

To show the sharpness of the constant factor

$$
\frac{B}{C}=\frac{|b(1-k)(\delta+2)+2|+|b(1-2 \alpha+k)(\delta+2)+2|}{2[2|b(1-\alpha)|+|b(1-k)(\delta+2)+2|+|b(1-2 \alpha+k)(\delta+2)+2|]},
$$


we consider the function:

$$
f_{1}(z)=z-\frac{2|b(1-\alpha)|}{B} z^{2}=\frac{B z-2 \mid b(1-\alpha) z^{2}}{B}
$$

$(z \in U ; \delta \geq-1 ; 0 \leq k \leq 1 ; b \in \mathbb{C} \backslash\{0\})$. Applying (1.10) with $g(z)=\frac{z}{1-z}$ and $f(z)=f_{1}(z)$ we have

$$
\frac{B z-2 b(\alpha-1) z^{2}}{C} \prec \frac{z}{1-z} .
$$

Using the fact that

$$
|\operatorname{Re} z| \leq|z|
$$

we now show that

$$
\min \left\{\operatorname{Re} \frac{B z-2 b(\alpha-1) z^{2}}{C}: z \in U\right\}=-\frac{1}{2} .
$$

Now,

$$
\begin{aligned}
\left|\operatorname{Re} \frac{B z-2|b(1-\alpha)| z^{2}}{C}\right| & \leq\left|\frac{B z-2|b(1-\alpha)| z^{2}}{C}\right| \\
& =\frac{|B z-2| b(1-\alpha)\left|z^{2}\right|}{|C|} \\
& \leq \frac{B|z|+2|b(1-\alpha)|\left|z^{2}\right|}{C} \\
& =\frac{B+2|b(1-\alpha)|}{C}=\frac{1}{2}
\end{aligned}
$$

$(|z|=1)$. This implies that

$$
\left|\operatorname{Re} \frac{B z-2|b(1-\alpha)| z^{2}}{C}\right| \leq \frac{1}{2}
$$

i.e.,

$$
-\frac{1}{2} \leq \operatorname{Re} \frac{B z-2|b(1-\alpha)| z^{2}}{C} \leq \frac{1}{2}
$$

Hence,

$$
\min \left\{\operatorname{Re}\left(\frac{B}{C} f_{1}(z)\right): z \in U\right\}=-\frac{1}{2},
$$

which completes the proof of Theorem 1.3. 
3. Some applications. Taking $\delta=1$ and $b=1$ in Theorem 1.3, we obtain the following:

Corollary 1. If the function $f(z)$ defined by $(1.1)$ is in $M^{\delta^{*}}(b, \alpha)$, then

$$
\frac{|5-3 \alpha|}{2|6-4 \alpha|}(f * g)(z) \prec g(z)
$$

$(z \in U ; \alpha>1, g \in K(\alpha))$. In particular,

$$
\operatorname{Re}(f(z))>-\frac{|6-4 \alpha|}{|5-3 \alpha|}
$$

The constant factor

$$
\frac{|5-3 \alpha|}{2|6-4 \alpha|}
$$

cannot be replaced by any larger one.

Remark 1. By taking $\alpha=\frac{71}{45}>1$ in Corollary 1, we obtain the result of Aouf et al. [1]

Taking $b=1, \delta=0$ in Theorem 1.3, we obtain the following:

Corollary 2. If the function $f(z)$ defined by $(1.1)$ is in $M^{\delta^{*}}(b, \alpha)$, then

$$
\frac{|2-\alpha|}{|5-3 \alpha|}(f * g)(z) \prec g(z)
$$

$(z \in U ; \alpha>1, g \in K(\alpha))$. In particular,

$$
\operatorname{Re}(f(z))>-\frac{|5-3 \alpha|}{2|2-\alpha|}, \quad z \in U
$$

The constant factor

$$
\frac{|2-\alpha|}{|5-3 \alpha|}
$$

cannot be replaced by any larger one.

Remark 2. By taking $\alpha=\frac{11}{6}$ and $\alpha=\frac{20}{11}$ in Corollary 2, we obtain the results of Selvaraj and Karthikeyan [4].

Taking $b=1, \delta=-1$ and $k=0$ in Theorem 1.3, we obtain the following:

Corollary 3. If the function $f(z)$ defined by $(1.1)$ is in $M^{\delta^{*}}(b, \alpha)$, then

$$
\frac{|3-\alpha|}{|8-4 \alpha|}(f * g)(z) \prec g(z)
$$

$(z \in U ; \alpha>1, g \in K(\alpha))$. In particular,

$$
\operatorname{Re}(f(z))>-\frac{|4-2 \alpha|}{|3-\alpha|}, \quad z \in U .
$$


The constant factor

$$
\frac{|3-\alpha|}{|8-4 \alpha|}
$$

cannot be replaced by any larger one.

Remark 3. If we take $\alpha=\frac{7+3 m}{3+m}$ in Corollary $3,(m>0)$ and in particular $m=1$ (i.e., $\alpha=\frac{5}{2}>1$ ), we obtain the result of Attiya et al. [2].

\section{REFERENCES}

[1] Aouf, M. K., Shamandy, A., Mostafa, A. O., El-Emam, F., Subordination results associated with $\beta$-uniformly convex and starlike functions, Proc. Pakistan Acad. Sci. 46, no. 2 (2009), 97-101.

[2] Attiya, A. A., Cho, N. E., Kutbi, M. A., Subordination properties for certain analytic functions, Int. J. Math. Math. Sci. 2008, Article ID 63825 (2008).

[3] Latha, S., Shivarudrappa, L., A note on coefficient estimates for a class of analytic functions, Advances in Inequalities for Series (2008), 143-149.

[4] Selvaraj, C., Karthikeyan, K. R., Certain subordination results for a class of analytic functions defined by the generalized integral operator, Int. J. Comput. Math. Sci. 2, no. 4 (2008), 166-169.

[5] Wilf, H. S., Subordinating factor sequences for some convex maps of unit circle, Proc. Amer. Math. Soc. 12 (1961), 689-693.

E. A. Oyekan

Department of Mathematics and Statistics

Bowen University

Iwo, Osun State

Nigeria

e-mail: shalomfa@yahoo.com
T. O. Opoola

Department of Mathematics

University of Ilorin

Ilorin

Nigeria

e-mail: opoolato@unilorin.edu.ng

Received August 8, 2011 